\title{
Effect of switching from natalizumab to moderate- vs high-efficacy DMT in clinical practice
}

Carrie M. Hersh, DO, Haleigh Harris, BA, Devon Conway, MD, and Le H. Hua, MD

Neurology: Clinical Practice Month 2020 vol. 00 no. 00 1-13 doi:10.1212/CPJ.0000000000000809
Correspondence

Carrie M. Hersh hershc@ccf.org

\begin{abstract}
Objective

To assess the real-world comparative effectiveness of switching from natalizumab (NTZ) to a moderate-efficacy (Mod) disease-modifying therapy (DMT) vs high-efficacy therapy (HET) in patients with multiple sclerosis (MS).
\end{abstract}

\section{Methods}

Patients discontinuing NTZ at two MS centers $(\mathrm{n}=556)$ who switched to Mod DMT ( $\mathrm{n}=270)$ vs HET $(n=130)$ were assessed using propensity score (PS) weighting. PS model covariates included demographics and baseline clinical and MRI characteristics. All outcomes were reported as Mod DMT vs HET.

\section{Results}

Of the patients included in the study, $48.6 \%$ switched to Mod DMT (dimethyl fumarate, $\mathrm{n}=$ 130; fingolimod, $\mathrm{n}=140$ ) vs $23.4 \%$ who switched to HET (ocrelizumab, $\mathrm{n}=106$; rituximab, $\mathrm{n}=17$; alemtuzumab, $\mathrm{n}=7$ ). Within the first 6 months post-NTZ, switchers to Mod DMT experienced comparable relapses (odds ratio $[\mathrm{OR}]=1.36,95 \%$ confidence interval $[\mathrm{CI}]$ $[0.72-1.66], p=0.724)$, although they had increased MRI activity on treatment $(\mathrm{OR}=2.59$, 95\% CI [1.09-3.57], $p=0.037)$. By 24 months post-NTZ, there was no difference in the annualized relapse rate $(\mathrm{OR}=1.44,95 \% \mathrm{CI}[0.69-1.59], p=0.334)$ or time to first clinical relapse $(\mathrm{HR}=2.12,95 \% \mathrm{CI}[0.87-5.17], p=0.090)$, although switchers to Mod DMT had higher gadolinium-enhancing (GdE) lesions ( $\mathrm{OR}=3.62$, 95\% CI [1.56-5.21], $p=0.005$ ), earlier time to first $\mathrm{GdE}$ lesion ( $\mathrm{HR}=6.67,95 \% \mathrm{CI}[2.06-9.16], p=0.002)$, lower proportion with the absence of disease activity ( $\mathrm{OR}=0.41,95 \% \mathrm{CI}[0.21-0.71], p=0.004)$, and higher risk of disability progression on T25FW ( OR $=1.83,95 \% \mathrm{CI}[1.06-3.02], p=0.043)$ and 9HPT $(\mathrm{OR}=1.81,95 \%$ CI $[1.05-3.56], p=0.044)$.

\section{Conclusion}

Patients switching from NTZ to Mod DMT vs HET were at relatively increased risk of disease activity within the first 6 months of NTZ withdrawal that was sustained at 24 months, yielding greater disability progression. 
Natalizumab (NTZ) is a high-efficacy disease-modifying therapy (DMT) for relapsing forms of multiple sclerosis (MS). ${ }^{1-3}$ In clinical practice, long-term use of NTZ is limited by potential safety risks, including progressive multifocal leukoencephalopathy (PML), 4,5 that can be reduced by switching to a different DMT. However, the transition between NTZ and alternative therapies poses additional risks because disease reactivation can occur upon NTZ discontinuation in the form of relapses and/or gadolinium-enhancing (GdE) lesions. ${ }^{6-9}$ The risk of rebound disease-activity that is worse compared to the pre-NTZ period-increases with longer NTZ washout periods $>3$ months $^{10,11}$ and by switching to an alternative therapy with lower efficacy. ${ }^{12}$ Many studies investigated the effect of switching from NTZ to a different DMT in high-risk PML populations. ${ }^{10-23}$ However, consensus is still lacking in regard to DMT sequencing following NTZ cessation. A paucity of data is available that directly compare disease activity head to head across different DMT switching paradigms.

Real-world studies are therefore needed to investigate the effects of various post-NTZ sequencing strategies on both early and longer-term MS disease activity and disability progression. Published data demonstrated that patients de-escalating therapy from NTZ to a lower-efficacy DMT (e.g., glatiramer acetate, interferon beta, and teriflunomide) are at risk of rebound or breakthrough disease activity. ${ }^{12,18,20}$ However, patients may fare better by switching to a moderate DMT (Mod DMT) or high-efficacy therapy (HET).

We sought to fill an important knowledge gap by comparing the effectiveness of switching from NTZ to a Mod DMT vs another HET on MS outcomes in a clinical practice cohort at 6 months and 24 months using propensity score (PS)-adjusted methods. ${ }^{24}$ In the present study, we defined Mod DMT as treatment with either fingolimod or dimethyl fumarate, based on equivalent clinical and MRI outcome measures derived from various comparative effectiveness studies. ${ }^{25-30}$ We defined HET as treatment switch to ocrelizumab, rituximab, or alemtuzumab.

\section{Methods}

\section{Standard protocol approvals, registrations, and patient consents}

This study was approved by the Institutional Review Board at the Cleveland Clinic.

\section{Patient population}

We conducted a retrospective observational study of patients with MS treated at the Cleveland Clinic Lou Ruvo Center for Brain Health (LRCBH) and Cleveland Clinic Mellen Center who discontinued NTZ between December 2005 and January 2018 and switched either to a Mod DMT or another HET. Patients were identified through the $\mathrm{TOUCH}^{\odot}$ prescribing program database. All patients included in the study were previously diagnosed with MS by a Cleveland Clinic MS specialist at the LRCBH or the Mellen Center.

\section{Data collection}

All patients who switched from NTZ to a Mod DMT or HET and had 6-month and 24-month data available were identified. Baseline and outcome data were collected from the electronic medical record (EMR). To adequately capture baseline disease characteristics important for determining treatment allocation between the two therapeutic paradigms, we included covariates derived from the EMR data in the 12 months before NTZ exposure and within 6 months before NTZ discontinuation. These data were confirmed by an MS clinician before they were included in the data set. The study adjusted for NTZ washout duration $>3$ months to lower the risk of disease rebound as a potential confounder.

Demographics and clinical, radiographic, laboratory, and patient-reported outcome (PRO) measures were collected from the EMR. Follow-up assessments (e.g., clinician visits, MRI frequency, and protocols) did not differ between the groups. Clinical relapses-identified retrospectively in the patients charts-were designated by the treating neurologist and defined as new or worsening MS symptoms lasting greater than 24 hours without a coexisting fever or illness. The timed 25-foot walk $(\mathrm{T} 25 \mathrm{FW})^{31}$ and 9-hole peg test $(9-\mathrm{HPT})^{32}$ were measured by the treating health care providers as part of routine clinical practice. The number of new T2-hyperintense and GdE brain MRI lesions were manually counted by Cleveland Clinic neuroradiologists. Neuroradiologic data at 6-month follow-up were compared with brain MRIs 6 months before NTZ discontinuation, and 24-month neuroradiologic data were compared with brain MRIs within 12 months of initiating the switched DMT. To assess the patient experience, PRO measures, such as the Patient Health Questionnaire-9 (PHQ-9) ${ }^{33}$ and Performance Scale, ${ }^{34}$ were also collected. We stored collated information in an encrypted database using a passwordprotected Excel Spreadsheet on secure Cleveland Clinic servers.

\section{Statistical analysis}

Data were imported for analysis into $\mathrm{R}$ version 3.5.2. ${ }^{35}$ The majority of covariates incorporated into the PS model were missing in $<10 \%$ of patients. However, some covariates (e.g., baseline MRI data and JC virus antibody status) were missing in $>10 \%$ of patients. We used the same approach as in our previously conducted studies to account for missingness patterns in the PS. ${ }^{25,27}$

Analyses were conducted in both an intention-to-treat (ITT) model and on-treatment ("per protocol") as a sensitivity analysis for those switching from NTZ to their respective DMT strategy (Mod DMT or HET) by 6- and 24-month follow-up. The primary outcome was the annualized relapse rate ratio (ARR ratio; Mod DMT vs HET) at 24 months. The ARR was calculated by dividing the total number of relapses by the total number of person-years at risk. Secondary outcome measures included the time to first relapse, time to first GdE lesion, and proportions with new T2-hyperintense lesions, GdE lesions, MRI disease activity (a composite outcome measure defined as the proportion of patients with new T2-hyperintense lesions 
and/or GdE lesions), absence of disease activity (a composite outcome measure defined as freedom from clinical relapses and MRI disease activity), and 20\% worsening on the T25FW and 9HPT. $^{25,26,32}$

The PS was built as a logistic regression model to calculate the likeliness of switching from NTZ to Mod DMT, as opposed to HET, using a priori selected covariates (table 1). A PS was calculated for each patient and subsequently used in an Average Treatment effect on the Treated (ATT) weighting model to derive a sample of patients who were similar at baseline except for their allocated treatment paradigm. This approach reduced the effect of indication bias on our comparisons of NTZ switch to Mod DMT vs HET while still retaining information from all patients. Owing to different sample sizes across the two cohorts, a PS matching technique was not used because a large selection of patients would have remained unmatched, consequently introducing another selection bias into the PS model. ${ }^{24}$ Before deriving conclusions on treatment effect differences between our groups of interest, we selected PS weighting on the basis of a more complete balance across the variables included in our PS model between the two cohorts.

Similar to previous studies, ${ }^{25,27}$ the strength of the PS model was assessed by how well it balanced the two groups, as determined by comparing the standardized differences across the means of the covariates before and after PS adjustment. In this study, we determined excellent covariate balance as achieving an absolute standardized difference of $<10 \%$ on the means of the covariates across the two therapeutic strategies. Before ATT weighting, unadjusted outcome measures were estimated using Pearson $\chi^{2}$ tests for categorical data and $t$ tests for continuous data. After ATT weighting, conditional logistic regression models were used to calculate odds ratio (OR) estimates for binary outcomes and Cox proportional hazards models and Kaplan-Meier survival curves to obtain survival end points. The ARR was analyzed using a Poisson regression model. Odds and hazards ratios were measured as those who switched from NTZ to a Mod DMT compared with patients who switched to HET. The primary outcome measure (ARR ratio) was based on a twotailed test of statistical significance with $\alpha=0.05$. Assuming $80 \%$ power with a total sample size of 400 patients, the minimum detectable effect size was determined to be 0.26 .

\section{Data availability}

Anonymized data can be shared at the request of qualified investigators for purposes of replicating procedures and results.

\section{Results}

\section{Baseline characteristics}

In the original cohort, 556 patients from the Cleveland Clinic LRCBH and the Mellen Center discontinued NTZ between December 2005 and January 2018 (figure 1). The majority of patients withdrew from NTZ due to increased PML risks ( $\mathrm{n}$ $=305,54.9 \%)$. Of those who had 24-month follow-up data available for review, a total of 270 patients switched to Mod
DMT (fingolimod $\mathrm{n}=140$, dimethyl fumarate $\mathrm{n}=130$ ), and 130 patients transitioned to HET (ocrelizumab $\mathrm{n}=104$, rituximab $\mathrm{n}=18$, and alemtuzumab $\mathrm{n}=18$ ).

Demographics and baseline disease characteristics are presented in table 1 . The majority of patients in our cohort had relapsing-remitting $\mathrm{MS}$ at the time of natalizumab exposure (Mod DMT $=95.9 \%$, HET $=95.2 \%)$. A higher proportion of patients discontinuing NTZ due to increased PML risks switched to Mod DMT (72.7\%) compared with HET (60.1\%) $(p=0.019)$, which was consistent with the increased proportion of patients who switched to Mod DMT (62.6\%) vs HET (47.0\%) due to JC virus antibody seropositivity at any time $(p=0.027)$. The mean washout duration between groups was similar and relatively short (Mod DMT $=1.4$ months, HET $=1.8$ months; $p=0.340)$, reducing the risk of disease reactivation that otherwise would have posed an additional confounder. Duration of NTZ treatment did not differ between our cohorts. As expected, the proportion with baseline disease activity, measured via relapses and new T2 and/or GdE lesions, before starting NTZ was numerically higher compared with disease activity while on NTZ (table 1).

\section{Propensity score model}

The PS model was built using demographics and baseline clinical, radiographic, laboratory, and PRO characteristics summarized in table 1 . Missing covariate data did not considerably change the overall balance of covariates after PS weighting. The model correctly assigned higher PS to the Mod DMT group compared with the HET group (figure e-1, links. lww.com/CPJ/A162), given that the logistic regression model was calculated to determine the likelihood of switching from NTZ to the former treatment paradigm. Before ATT weighting, the treatment groups were not balanced well with at least half of the covariates falling outside of the $10 \%$ absolute standardized difference range. Furthermore, the absolute value of the standardized difference of the linear PS comparing Mod DMT with HET was 91.2\%, which was considerably greater than the $50 \%$ standard previously recommended by Rubin. ${ }^{36}$ Therefore, we determined that PS adjustment was warranted to account for indication bias before making conclusions on differences in treatment effects.

PS weighting balanced the two treatment groups effectively with only 5 covariates obtaining absolute standardized differences $>10 \%$ (figure e-2, links.lww.com/CPJ/A162). In a double robust approach, PS weighting also produced a similar linear PS distribution with a standardized difference of $17.2 \%$, which was sufficiently within the $50 \%$ standard.

\section{Outcome estimates by 6-month follow-up}

To estimate the effectiveness of switching from NTZ to a Mod DMT vs HET on early MS disease activity, we compared inflammatory disease outcomes (e.g., relapse, MRI activity, and absence of disease activity) within 6 months of NTZ discontinuation. Unadjusted and postweighting outcomes are summarized in tables 2 and 3. By 6 months, $11.7 \%$ of Mod 
Table 1 Baseline characteristics of NTZ switchers to Mod DMT vs HET ${ }^{a}$

\begin{tabular}{|c|c|c|c|c|c|}
\hline & \multicolumn{2}{|c|}{ Moderate efficacy, $n=270$} & \multicolumn{2}{|c|}{ High efficacy, $n=130$} & \multirow[b]{2}{*}{$p$ Value } \\
\hline & $\mathrm{n}$ or mean & $\%$ or SD & $\mathrm{n}$ or mean & $\%$ or SD & \\
\hline \multicolumn{6}{|l|}{ Demographics } \\
\hline Age at diagnosis (years, SD) & 33.3 & 9.0 & 32.1 & 9.6 & 0.228 \\
\hline $\begin{array}{l}\text { Age at NTZ discontinuation } \\
\text { (years, SD) }\end{array}$ & 42.5 & 9.9 & 42.2 & 10.8 & 0.778 \\
\hline Sex (female) & 182 & $67.4 \%$ & 88 & $67.7 \%$ & 1.000 \\
\hline Race & & & & & 0.079 \\
\hline White & 224 & $86.2 \%$ & 92 & $75.4 \%$ & \\
\hline Black & 32 & $12.3 \%$ & 26 & $21.3 \%$ & \\
\hline Other & 4 & $1.6 \%$ & 4 & $3.3 \%$ & \\
\hline \multicolumn{6}{|l|}{ Comorbidities } \\
\hline Tobacco smoking & 72 & $26.7 \%$ & 29 & $22.3 \%$ & 0.414 \\
\hline Asthma & 27 & $10.0 \%$ & 17 & $13.1 \%$ & 0.072 \\
\hline Cancer & 11 & $4.1 \%$ & 1 & $0.8 \%$ & 0.133 \\
\hline COPD & 4 & $1.5 \%$ & 0 & $0.0 \%$ & 0.391 \\
\hline Diabetes mellitus & 24 & $8.9 \%$ & 8 & $6.2 \%$ & 0.455 \\
\hline Hyperlipidemia & 45 & $16.7 \%$ & 10 & $7.7 \%$ & $0.022^{\mathrm{b}}$ \\
\hline Hypertension & 57 & $21.1 \%$ & 17 & $13.1 \%$ & 0.072 \\
\hline \multicolumn{6}{|l|}{ Disease history } \\
\hline Disease duration (years, SD) & 17.1 & 8.6 & 15.1 & 8.9 & $0.041^{b}$ \\
\hline Relapsing-remitting MS at disease onset & 255 & $95.9 \%$ & 120 & $95.2 \%$ & 0.422 \\
\hline Disease course at NTZ discontinuation & & & $0.021^{b}$ & & \\
\hline Relapsing-remitting & 199 & $74.8 \%$ & 109 & $83.8 \%$ & \\
\hline Primary progressive & 2 & $0.8 \%$ & 3 & $2.3 \%$ & \\
\hline Progressive relapsing & 10 & $3.8 \%$ & 0 & $0.0 \%$ & \\
\hline Secondary progressive & 55 & $20.7 \%$ & 18 & $13.8 \%$ & \\
\hline No. of previous DMTs (number, SD) & 2.9 & 1.6 & 3.0 & 1.5 & 0.443 \\
\hline Interferon & 229 & $84.8 \%$ & 95 & $73.1 \%$ & $0.037^{b}$ \\
\hline Glatiramer & 115 & $42.6 \%$ & 55 & $42.3 \%$ & 1.000 \\
\hline Fingolimod & 10 & $3.7 \%$ & 23 & $17.7 \%$ & $<0.001^{\mathrm{b}}$ \\
\hline Dimethyl fumarate & 6 & $2.2 \%$ & 31 & $24.0 \%$ & $<0.001^{\mathrm{b}}$ \\
\hline Teriflunomide & 2 & $0.7 \%$ & 5 & $3.8 \%$ & 0.070 \\
\hline Mitoxantrone & 6 & $2.2 \%$ & 0 & $0.0 \%$ & 0.203 \\
\hline Other immunosuppressive & 30 & $11.1 \%$ & 10 & $5.9 \%$ & 0.065 \\
\hline NTZ infusions, mean (range) & $35(1-93)$ & N/A & $28(1-65)$ & $\mathrm{N} / \mathrm{A}$ & 0.142 \\
\hline \multicolumn{6}{|l|}{ Reason for NTZ discontinuation } \\
\hline Breakthrough disease & 40 & $14.8 \%$ & 24 & $18.5 \%$ & 0.776 \\
\hline Intolerance & 53 & $19.6 \%$ & 31 & $23.8 \%$ & 0.504 \\
\hline NTZ risks (PML) & 196 & $72.7 \%$ & 78 & $60.1 \%$ & $0.019^{\mathrm{b}}$ \\
\hline
\end{tabular}


Table 1 Baseline characteristics of NTZ switchers to Mod DMT vs HET'a (continued)

\begin{tabular}{|c|c|c|c|c|c|}
\hline & \multicolumn{2}{|c|}{ Moderate efficacy, $n=270$} & \multicolumn{2}{|c|}{ High efficacy, n = 130} & \multirow[b]{2}{*}{$p$ Value } \\
\hline & $\mathrm{n}$ or mean & $\%$ or SD & $\mathrm{n}$ or mean & $\%$ or SD & \\
\hline DMT washout (mean, SD) & 1.4 & 5.5 & 1.8 & 4.5 & 0.340 \\
\hline \multicolumn{6}{|l|}{ Labs } \\
\hline \multicolumn{6}{|l|}{$\begin{array}{l}\text { JCV antibody status at NTZ } \\
\text { discontinuation }\end{array}$} \\
\hline Negative & 68 & $35.8 \%$ & 59 & $50.4 \%$ & $0.027^{\mathrm{b}}$ \\
\hline Positive & 119 & $62.6 \%$ & 55 & $47.0 \%$ & \\
\hline Indeterminate & 3 & $1.6 \%$ & 3 & $2.6 \%$ & \\
\hline $\begin{array}{l}\text { ALC at NTZ discontinuation } \\
\left(\times 10^{9} / \mathrm{L} ; \text { mean, SD }\right)\end{array}$ & 3.2 & 1.1 & 3.2 & 1.0 & 0.739 \\
\hline \multicolumn{6}{|l|}{ Disease activity } \\
\hline Relapse before NTZ initiation & 103 & $40.1 \%$ & 56 & $48.3 \%$ & 0.171 \\
\hline Relapse during NTZ treatment & 13 & $4.8 \%$ & 7 & $5.4 \%$ & 1.000 \\
\hline Brain MRI before NTZ initiation & 229 & $84.8 \%$ & 100 & $76.9 \%$ & \\
\hline GdE lesions & 102 & $44.5 \%$ & 46 & $46.0 \%$ & 0.901 \\
\hline New T2 lesions & 85 & $37.1 \%$ & 45 & $46.9 \%$ & 0.130 \\
\hline $\begin{array}{l}\text { Brain MRI } 6 \text { months before NTZ } \\
\text { discontinuation }\end{array}$ & 96 & $35.6 \%$ & 64 & $49.2 \%$ & \\
\hline GdE lesions & 3 & $3.2 \%$ & 0 & $0.0 \%$ & 0.436 \\
\hline New T2 lesions & 2 & $2.1 \%$ & 0 & $0.0 \%$ & 0.663 \\
\hline \multicolumn{6}{|l|}{ Objective measures } \\
\hline T25FW (s, SD) & 9.1 & 9.0 & 7.3 & 3.9 & 0.086 \\
\hline Ambulation assistance & & & & 0.175 & \\
\hline None & 174 & $69.9 \%$ & 88 & $79.3 \%$ & \\
\hline Unilateral & 26 & $10.4 \%$ & 8 & $7.2 \%$ & \\
\hline Bilateral & 38 & $15.3 \%$ & 9 & $8.1 \%$ & \\
\hline Wheelchair & 11 & $4.4 \%$ & 6 & $5.4 \%$ & \\
\hline 9-HPT dominant (mean sec, SD) & 30.5 & 16.9 & 26.4 & 9.3 & 0.099 \\
\hline \multicolumn{6}{|l|}{ Patient-reported outcomes } \\
\hline MS performance scale & 15.0 & 7.7 & 14.5 & 8.5 & 0.694 \\
\hline PHQ-9 score, depressed & 69 & $43.7 \%$ & 27 & $38.0 \%$ & 0.512 \\
\hline $\begin{array}{l}\text { Abbreviations: 9-HPT = 9-hole peg te } \\
\text { moderate-efficacy DMT; NTZ = natali } \\
\text { NTZ discontinuation due to breakthr } \\
\text { PHQ-9 score, depressed defined as P } \\
\text { a Unless otherwise specified in the ta } \\
\text { b Statistically significant } p \text { value (alph }\end{array}$ & $\begin{array}{l}\text { lymphocyte c } \\
\text { atient Health } \\
\text { fined as NTZ } \\
\text { se characteri }\end{array}$ & $\begin{array}{l}\text { gadoliniur } \\
\text { ire-9; T25F } \\
\text { le to clinic } \\
\text { as the tin }\end{array}$ & $\begin{array}{l}\text {; } \mathrm{HET}=\text { high- } \\
5 \text {-foot walk; } \\
\text { ew MRI activ } \\
\text { thin } 12 \text { mont }\end{array}$ & $\begin{array}{l}\text { erapy; JCV } \\
\text { iple sclero } \\
\text { gression } \\
\text { initiation. }\end{array}$ & ; Mod = \\
\hline
\end{tabular}

DMT-treated patients experienced a clinical relapse vs $8.7 \%$ of HET-treated patients with a PS-adjusted OR $=1.36(95 \%$ confidence interval $[\mathrm{CI}][0.72-1.66], p=0.724)$. In an ITT model, MRI disease activity favored those who switched to HET $(\mathrm{OR}=1.68, p=0.068)$ but did not reach statistical significance. In a sensitivity analysis including only patients on treatment, those treated with Mod DMT had higher MRI disease activity $(\mathrm{OR}=2.59,95 \% \mathrm{CI}[1.09-3.87], p=0.037)$, GdE lesions ( $\mathrm{OR}=3.37,95 \% \mathrm{CI}[1.45-4.68], p=0.007)$, and new T2-hyperintense lesions ( $\mathrm{OR}=2.18,95 \% \mathrm{CI}$ [1.10-4.01], $p=0.029)$. Furthermore, of those who had available clinical and MRI data by 6-month follow-up (Mod DMT $\mathrm{n}=238$, HET $\mathrm{n}=$ 109), patients switching from NTZ to Mod DMT had lower likelihood of achieving absence of disease activity (61.3\%) 


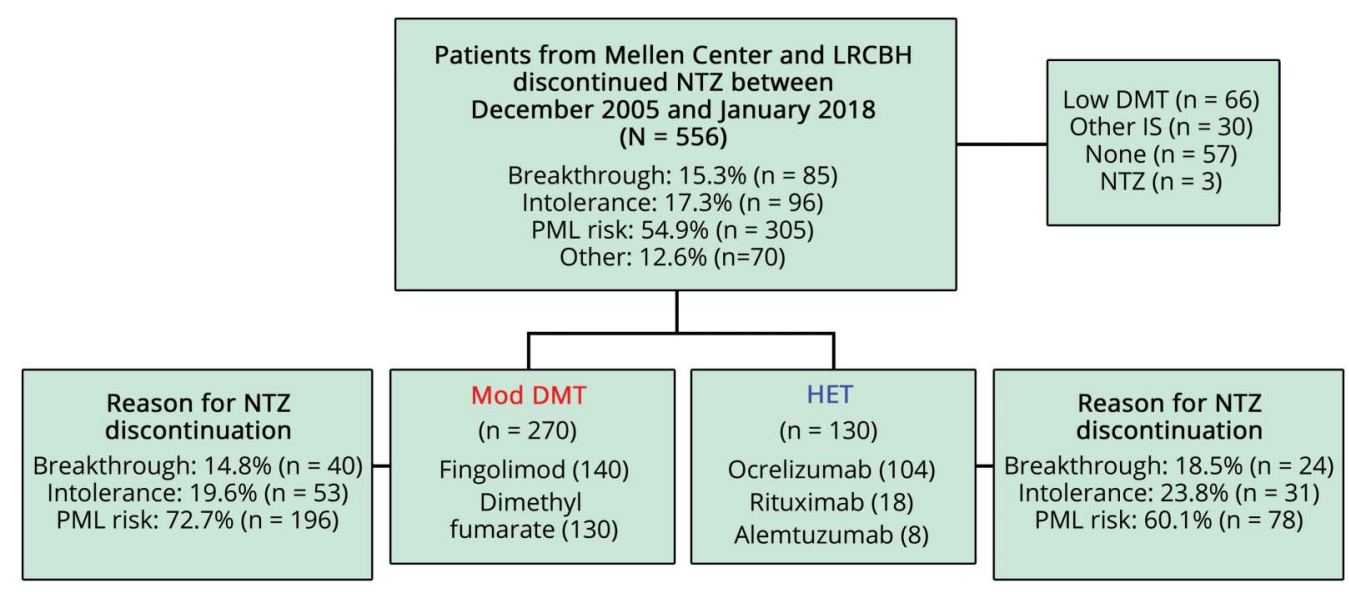

At 24-month follow-up, 270 patients switched from NTZ to a Mod DMT (fingolimod and dimethyl fumarate), and 130 patients switched to HET (ocrelizumab, rituximab, and alemtuzumab). The majority of patients discontinued NTZ due to increased PML risks, of which a greater proportion switched to Mod DMT vs HET. Low (efficacy) DMT: glatiramer acetate, interferon beta, teriflunomide. Other IS: azathioprine, mycophenolate mofetil, cyclophosphamide, pulse methylprednisolone, and pulse IVIG. DMT = disease-modifying therapy; HET = high-efficacy therapy; IS = immunosuppressive therapy; Mod = moderateefficacy DMT; NTZ = natalizumab; PML = progressive multifocal leukoencephalopathy.

compared with those who switched to HET (80.7\%) (OR = 0.42 , 95\% CI [0.23-0.76], $p=0.004)$.

\section{Outcome estimates by 24-month follow-up}

To determine the durability of treatment effect on inflammatory disease activity and disability progression, we assessed 24month outcomes in NTZ switchers to Mod DMT vs HET (tables 2 and 3). By 24 months, 13.3\% of Mod DMT patients had a clinical relapse vs $10.0 \%$ of HET patients. Mod DMT patients had 36 relapses over 171.4 patient-years of treatment with $\mathrm{ARR}=0.21$ (95\% CI = 0.07-0.39). HET patients had 13 relapses over 118.2 patient-years of treatment with $\mathrm{ARR}=0.11$ (95\% CI $=0.05-0.42)$. After PS weighting, there was no difference in the ARR (ARR ratio = 1.44, 95\% CI [0.69-1.59], $p=$ 0.334). The mean time to first relapse was 3.2 months for Mod DMT compared with 4.4 months for HET. Although survival outcomes favored HET, no difference was found in time to first relapse between groups over the first 24 months of treatment $(\mathrm{HR}=2.12,95 \% \mathrm{CI}[0.87-5.17], p=0.090)$ (figure 2).

In an ITT model, among all patients with available MRI data by 24-month follow-up (Mod DMT $\mathrm{n}=252$, HET $\mathrm{n}=126$ ), $25.4 \%$ of patients on Mod DMT demonstrated MRI activity vs $11.9 \%$ on HET $(\mathrm{OR}=2.43,95 \%$ CI [1.08-3.93], $p=0.039)$. A sensitivity analysis comparing only patients on treatment showed similar findings. Furthermore, patients treated with Mod DMT had higher odds of GdE lesions (OR = 1.99, 95\% CI [1.12-2.73], $p=0.022)$ and new T2-hyperintense lesions $(\mathrm{OR}=2.15,95 \%$ CI $[1.18-3.01], p=0.011)$ with similar findings in a sensitivity analysis only comparing patients on treatment (tables 2 and 3). Patients who switched to Mod DMT also experienced earlier time to first GdE lesion compared with HET (HR $=6.67,95 \%$ CI [2.06-9.16), $p=0.002)$ (figure 3). An additional sensitivity analysis comparing inflammatory disease outcomes stratified by reason for discontinuation - breakthrough disease and safety risks - also showed similar results to the entire cohort, although comparative increased disease activity in the Mod DMT group appeared to be driven by NTZ switchers due to breakthrough disease (table 4).

Of patients with available on-treatment clinical and MRI outcome data by 24 months (Mod DMT n = 127, HET n = 92), NTZ switchers to Mod DMT had lower likelihood of absence of disease activity (63.7\%) vs HET (80.4\%) (OR $=0.41,95 \%$ CI $[0.21-0.71], p=0.004)$. Using surrogate markers of disability progression, NTZ switchers to Mod DMT experienced higher odds of $20 \%$ worsening of the T25FW (OR $=1.83,95 \%$ CI [1.06-3.02], $p=0.043$ ) and 20\% worsening of the 9-HPT $(\mathrm{OR}=1.81,95 \% \mathrm{CI}[1.05-3.56], p=0.044)$. There were no cases of PML or other serious opportunistic infections reported in either cohort.

\section{Discussion}

NTZ is a high-efficacy DMT indicated for relapsing forms of MS, for which long-term use in clinical practice is limited by potential safety risks, including PML. The risk is particularly high in patients who (1) have a high anti-JC virus antibody index, (2) had NTZ exposure longer than 24 months, and (3) had previous exposure to immunosuppressive therapies. ${ }^{4,5}$ In the current neurotherapeutic landscape of multiple available DMTs with various mechanisms of action and effectiveness and safety profiles, it is possible to lower these PML risks by switching patients to another therapy. However, an additional risk of disease reactivation occurs upon NTZ interruption. After NTZ discontinuation, the time to return of $\alpha 4 \beta 1$-integrin antigen saturation to values found in untreated patients likely 
Table 2 Summary of unadjusted effectiveness outcomes of NTZ switchers to Mod DMT VS HET

\begin{tabular}{|c|c|c|c|c|c|}
\hline & \multicolumn{2}{|c|}{ Moderate efficacy, n = 270} & \multicolumn{2}{|c|}{ High efficacy, n = 130} & \multirow[b]{2}{*}{$p$ Value } \\
\hline & $\mathrm{n}$ & $\%$ or SD & $\mathrm{n}$ & $\%$ or SD & \\
\hline \multicolumn{6}{|l|}{ 6-month outcomes } \\
\hline Clinical relapse (no. of patients) & 31 & $11.7 \%$ & 11 & $8.7 \%$ & 0.462 \\
\hline Relapses per patient (mean, SD) & 0.12 & 0.32 & 0.09 & 0.28 & 0.364 \\
\hline $\begin{array}{l}\text { MRI available for review } \\
\text { (intention to treat) }\end{array}$ & 219 & $81.1 \%$ & 107 & $82.3 \%$ & 0.824 \\
\hline MRI activity (GdE + new T2 lesions) & 47 & $21.5 \%$ & 12 & $11.2 \%$ & 0.054 \\
\hline GdE lesions & 37 & $16.9 \%$ & 11 & $10.3 \%$ & 0.285 \\
\hline New T2 lesions & 46 & $21.0 \%$ & 15 & $14.0 \%$ & 0.127 \\
\hline MRI available for review (on treatment) & 184 & $68.1 \%$ & 94 & $72.6 \%$ & 0.133 \\
\hline MRI activity (GdE + new T2 lesions) & 46 & $25.0 \%$ & 8 & $8.5 \%$ & $0.039^{b}$ \\
\hline GdE lesions & 30 & $16.3 \%$ & 2 & $2.1 \%$ & $0.014^{b}$ \\
\hline New T2 lesions & 39 & $21.4 \%$ & 8 & $8.5 \%$ & $0.042^{b}$ \\
\hline Absence of disease activity ${ }^{a}$ & $146 / 238$ & $61.3 \%$ & $88 / 109$ & $80.7 \%$ & $0.005^{\mathrm{b}}$ \\
\hline \multicolumn{6}{|l|}{ 24-month outcomes } \\
\hline Clinical relapse (no. of patients) & 36 & $13.3 \%$ & 13 & $10.0 \%$ & 0.122 \\
\hline Relapses per patient (mean, SD) & 0.14 & 0.28 & 0.06 & 0.26 & 0.364 \\
\hline $\begin{array}{l}\text { MRI available for review } \\
\text { (intention to treat) }\end{array}$ & 252 & $93.3 \%$ & 126 & $96.9 \%$ & 0.244 \\
\hline MRI activity (GdE + new T2 lesions) & 64 & $25.4 \%$ & 15 & $11.9 \%$ & $0.012^{\mathrm{b}}$ \\
\hline GdE lesions & 44 & $17.5 \%$ & 11 & $8.7 \%$ & $0.028^{\mathrm{b}}$ \\
\hline New T2 lesions & 72 & $28.6 \%$ & 19 & $15.1 \%$ & $0.021^{b}$ \\
\hline MRI available for review (on treatment) & 136 & $50.4 \%$ & 81 & $62.3 \%$ & $0.011^{\mathrm{b}}$ \\
\hline MRI activity (GdE + new T2 lesions) & 26 & $19.1 \%$ & 8 & $9.9 \%$ & $0.029^{b}$ \\
\hline GdE lesions & 24 & $17.6 \%$ & 6 & $7.4 \%$ & $0.013^{b}$ \\
\hline New T2 lesions & 28 & $20.6 \%$ & 8 & $9.9 \%$ & $0.010^{\mathrm{b}}$ \\
\hline Absence of disease activity ${ }^{a}$ & $81 / 127$ & $63.7 \%$ & $74 / 92$ & $80.4 \%$ & $0.006^{\mathrm{b}}$ \\
\hline \multicolumn{6}{|l|}{ Measures of neurologic disability } \\
\hline T25FW (mean sec, SD) & 8.26 & 7.21 & 6.01 & 5.21 & $0.032^{b}$ \\
\hline $20 \%$ worsening of $\mathrm{T} 25 \mathrm{FW}$ & $113 / 203$ & $55.6 \%$ & $38 / 94$ & $40.2 \%$ & $0.045^{\mathrm{b}}$ \\
\hline 9-HPT dominant (mean sec, SD) & 24.93 & 10.65 & 21.36 & 10.11 & $0.039^{b}$ \\
\hline $20 \%$ worsening of $9-\mathrm{HPT}$ & $103 / 189$ & $54.5 \%$ & $37 / 83$ & $44.6 \%$ & $0.048^{b}$ \\
\hline
\end{tabular}

Abbreviations: 9-HPT = 9-hole peg test; DMT = disease-modifying therapy; GdE = gadolinium enhancing; HET = highly effective therapy; Mod = moderate; T25FW = timed 25-foot walk.

a Absence of disease activity defined as absence of clinical relapses + absence of MRI activity (GdE lesions and/or new T2 lesions).

${ }^{\mathrm{b}}$ Statistically significant $p$ value (alpha $=0.05$ ).

vary across individuals and is predictive of the risk of GdE lesion occurrence and rebound disease. , $^{87,38}$

Consensus is currently lacking on DMT switching strategies after NTZ withdrawal and is primarily based on single-arm investigations with variable outcomes, a few small comparative effectiveness studies, or expert opinion. Further observational studies are thus warranted to compare the effectiveness of postNTZ sequencing strategies to guide decision making in clinical practice. To fill this knowledge gap, we conducted a real-world PS-adjusted study comparing patients switching from NTZ to Mod DMT vs HET in a clinical practice cohort. We investigated 
Table 3 Unadjusted and adjusted effectiveness outcomes of NTZ switchers to Mod DMT vs HET

\begin{tabular}{|c|c|c|c|c|c|c|}
\hline & \multicolumn{3}{|l|}{ Unadjusted } & \multicolumn{3}{|c|}{ Propensity adjusted } \\
\hline & $\begin{array}{l}\text { Odds or } \\
\text { hazards ratio }\end{array}$ & $95 \% \mathrm{Cl}$ & $p$ Value & $\begin{array}{l}\text { Odds or } \\
\text { hazards ratio }\end{array}$ & $95 \% \mathrm{Cl}$ & $p$ Value \\
\hline \multicolumn{7}{|l|}{ 6-month outcomes } \\
\hline Proportion with clinical relapse & 1.32 & $0.66-2.03$ & 0.764 & 1.36 & $0.72-1.66$ & 0.724 \\
\hline \multicolumn{7}{|l|}{ MRI disease activity (intention-to-treat) } \\
\hline MRI activity (GdE + new T2 lesions) & 1.72 & $0.92-2.85$ & 0.077 & 1.68 & $0.94-2.13$ & 0.068 \\
\hline GdE lesions & 1.61 & $0.76-3.39$ & 0.281 & 1.63 & $0.83-2.69$ & 0.202 \\
\hline New T2 lesions & 1.57 & $0.81-3.05$ & 0.200 & 1.58 & $0.84-2.91$ & 0.189 \\
\hline \multicolumn{7}{|l|}{ MRI disease activity (on-treatment) } \\
\hline MRI activity (GdE + new T2 lesions) & 2.63 & $1.11-4.19$ & $0.032^{b}$ & 2.59 & $1.09-3.87$ & $0.037^{b}$ \\
\hline GdE lesions & 3.35 & $1.42-4.85$ & $0.006^{b}$ & 3.37 & $1.45-4.68$ & $0.007^{b}$ \\
\hline New T2 lesions & 2.14 & $1.08-4.12$ & $0.038^{b}$ & 2.18 & $1.10-4.01$ & $0.029^{b}$ \\
\hline Absence of disease activity ${ }^{a}$ & 0.52 & $0.34-0.82$ & $0.005^{\mathrm{b}}$ & 0.42 & $0.23-0.76$ & $0.004^{b}$ \\
\hline \multicolumn{7}{|l|}{ 24-month outcomes } \\
\hline Annualized relapse rate (ARR) ratio & 1.43 & $0.72-1.96$ & 0.423 & 1.44 & $0.69-1.59$ & 0.334 \\
\hline Proportion with clinical relapse & 1.40 & $0.68-2.88$ & 0.389 & 1.36 & $0.62-1.78$ & 0.711 \\
\hline Time to first relapse (months, mean) & 1.74 & $0.84-4.23$ & 0.126 & 2.12 & $0.87-5.17$ & 0.090 \\
\hline \multicolumn{7}{|l|}{ MRI disease activity (intent to treat) } \\
\hline MRI activity (GdE + new T2 lesions) & 2.36 & $1.07-4.12$ & $0.038^{b}$ & 2.43 & $1.08-3.93$ & $0.039^{b}$ \\
\hline GdE lesions & 1.89 & $1.08-3.24$ & $0.037^{b}$ & 1.99 & $1.12-2.73$ & $0.022^{b}$ \\
\hline New T2 lesions & 2.02 & $1.09-3.03$ & $0.018^{b}$ & 2.15 & $1.18-3.01$ & $0.011^{\mathrm{b}}$ \\
\hline \multicolumn{7}{|l|}{ MRI disease activity (on treatment) } \\
\hline MRI activity (GdE + new T2 lesions) & 2.72 & $1.16-3.13$ & $0.032^{b}$ & 2.54 & $1.18-3.07$ & $0.021^{\mathrm{b}}$ \\
\hline GdE lesions & 3.59 & $1.49-5.48$ & $0.006^{b}$ & 3.62 & $1.56-5.21$ & $0.005^{\mathrm{b}}$ \\
\hline New T2 lesions & 2.14 & $1.08-4.12$ & $0.033^{\mathrm{b}}$ & 2.23 & $1.17-3.88$ & $0.024^{b}$ \\
\hline Time to first GdE lesion (months, mean) & 5.23 & $2.12-8.64$ & $0.003^{b}$ & 6.67 & $2.06-9.16$ & $0.002^{\mathrm{b}}$ \\
\hline Absence of disease activity ${ }^{a}$ & 0.55 & $0.28-0.77$ & $0.004^{b}$ & 0.41 & $0.21-0.71$ & $0.004^{b}$ \\
\hline \multicolumn{7}{|l|}{ Measures of neurologic disability } \\
\hline $20 \%$ worsening of $\mathrm{T} 25 \mathrm{FW}$ & 1.78 & $1.03-3.21$ & $0.045^{\mathrm{b}}$ & 1.83 & $1.06-3.02$ & $0.043^{b}$ \\
\hline $20 \%$ worsening of 9 -HPT & 1.76 & $1.01-3.68$ & $0.047^{b}$ & 1.81 & $1.05-3.56$ & $0.044^{b}$ \\
\hline $\begin{array}{l}\text { Abbreviations: 9-HPT = 9-hole peg test; } \mathrm{Cl}=\mathrm{co} \\
\text { therapy; Mod = moderate; NTZ = natalizumab; } \\
\text { a Absence of disease activity defined as absen } \\
\text { b Statistically significant } p \text { value (alpha }=0.05 \text { ). }\end{array}$ & $\begin{array}{l}\text { nce interval; DM } \\
\mathrm{W}=\text { timed } 25-\mathrm{fo} \\
\text { clinical relapses }\end{array}$ & $\begin{array}{l}\text { sease-modif } \\
\text { lk. } \\
\text { ence of MRI }\end{array}$ & $\begin{array}{l}\text { herapy; G } \\
\text { ty (GdE le }\end{array}$ & $\begin{array}{l}\text { gadolinium enha } \\
\text { and/or new T2 I }\end{array}$ & ; & ective \\
\hline
\end{tabular}

6-month time points to determine the early comparative effectiveness of NTZ switch to one of two treatment strategies and 24-month time points to capture the durability of the new treatment's effect.

Overall, disease activity remained low at 6-month and 24month follow-up in both post-NTZ treatment arms, relative to the 12 -month pre-NTZ period. Numerically, there was a signal for increased disease activity post-NTZ across both groups relative to the time on treatment, but it did not supersede that of the pre-NTZ period. Therefore, in our population, patients did not experience rebound disease-putatively related to an immune reconstitution inflammatory syndrome-like phenomenon. ${ }^{39}$ Likely, the low disease activity was related to two different mechanisms in our study population: (1) a short washout duration (mean $<2$ months) across both treatment 


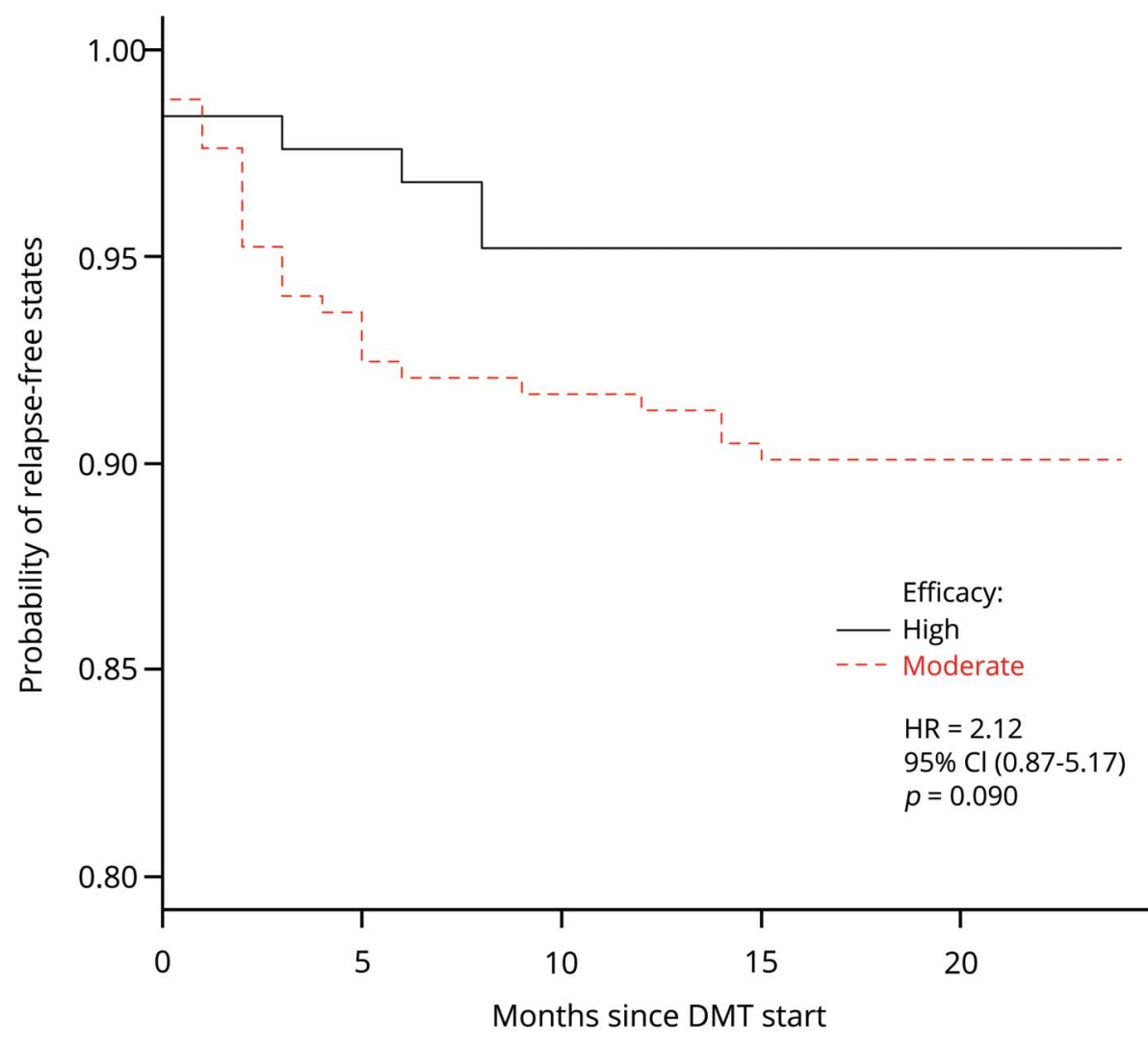

\begin{abstract}
Kaplan-Meier survival analysis showed a twofold higher hazard of first clinical relapse in NTZ switchers to moderate- vs high-efficacy DMT but did not reach statistical significance. DMT = disease-modifying therapy.
\end{abstract}

groups and (2) a form of inflammatory protection by switching to an alternative DMT of at least moderate efficacy. The French TYSEDMUS study $(n=4,055)$ showed that $37.1 \%$ of patients who discontinued NTZ experienced a relapse within 12 months of drug interruption, mainly occurring between 3 and 5 months of NTZ cessation. Similarly, this study did not observe rebound disease activity with a pre-NTZ ARR $=1.99$ and post$\mathrm{NTZ}$ ARR $=0.65 .^{9}$ On the other hand, a smaller study investigating the effects of NTZ interruption showed that the cumulative probability of rebound disease activity was 39\% and mostly occurred between 3 and 9 months after NTZ cessation, independently of an alternative DMT. ${ }^{6}$ These reported differences in relapse rates and risk of rebound disease upon NTZ cessation are likely related to variable use and sequencing of DMTs across centers and the restrictive availability of NTZ in France to patients with very active disease.

Various studies previously demonstrated the benefit of switching to an alternative DMT following NTZ discontinuation to reduce the risk of rebound disease. In 1 study, VillaverdeGonzalez et al. showed that $71.4 \%$ of patients remained relapse-free after switching from NTZ to one of the platform DMTs. Furthermore, $62.5 \%$ of patients experienced no radiologic activity 12 months after NTZ cessation. ${ }^{40}$ However, this study was limited by a small sample size and the use of less effective DMTs (e.g., glatiramer acetate and interferon beta) post-NTZ, which are suspected to have limitations in preventing rebound disease. ${ }^{19-21}$ Patients switching to more efficacious therapies may have better disease control. ${ }^{11,13-17,22,23}$ For instance, a large study $(\mathrm{n}=536)$ showed that patients switching from NTZ to fingolimod experienced only a small rise in the relapse rate $(A R R=0.38)$ relative to the period while on $\mathrm{NTZ}(\mathrm{ARR}=0.26, p=0.002) .{ }^{41}$

Our PS analysis demonstrated no differences in clinical relapses between NTZ switchers to Mod DMT vs HET, although it numerically favored HET. The mean time to first clinical relapse (3-5 months) observed in our patient population was consistent with the natural course of drug elimination and what was previously reported in a number of smaller studies. ${ }^{6,38} \mathrm{Al}-$ though there were no differences across early MRI disease activity end points in an ITT model, our sensitivity analysis ontreatment at 6-month follow-up and cumulative MRI disease activity over 24 months demonstrated lower effectiveness in NTZ switchers to Mod DMT compared with HET. Data in patients switching from NTZ to other HET are scarce. However, 1 comparative effectiveness study favored rituximab $(\mathrm{n}=$ $114)$ over fingolimod $(n=142)$ in patients discontinuing NTZ in relation to relapses (rituximab 1.8\%, fingolimod 17.6\%) and GdE lesions ( $0.9 \%$ vs $16.2 \%)$ at 1.5 years. ${ }^{22}$ These data are consistent with those reported in the current investigation. Another small observational study $(\mathrm{n}=16)$ investigating patients who switched from NTZ to alemtuzumab had no relapses, MRI activity, or increased EDSS scores. ${ }^{23}$ These data 


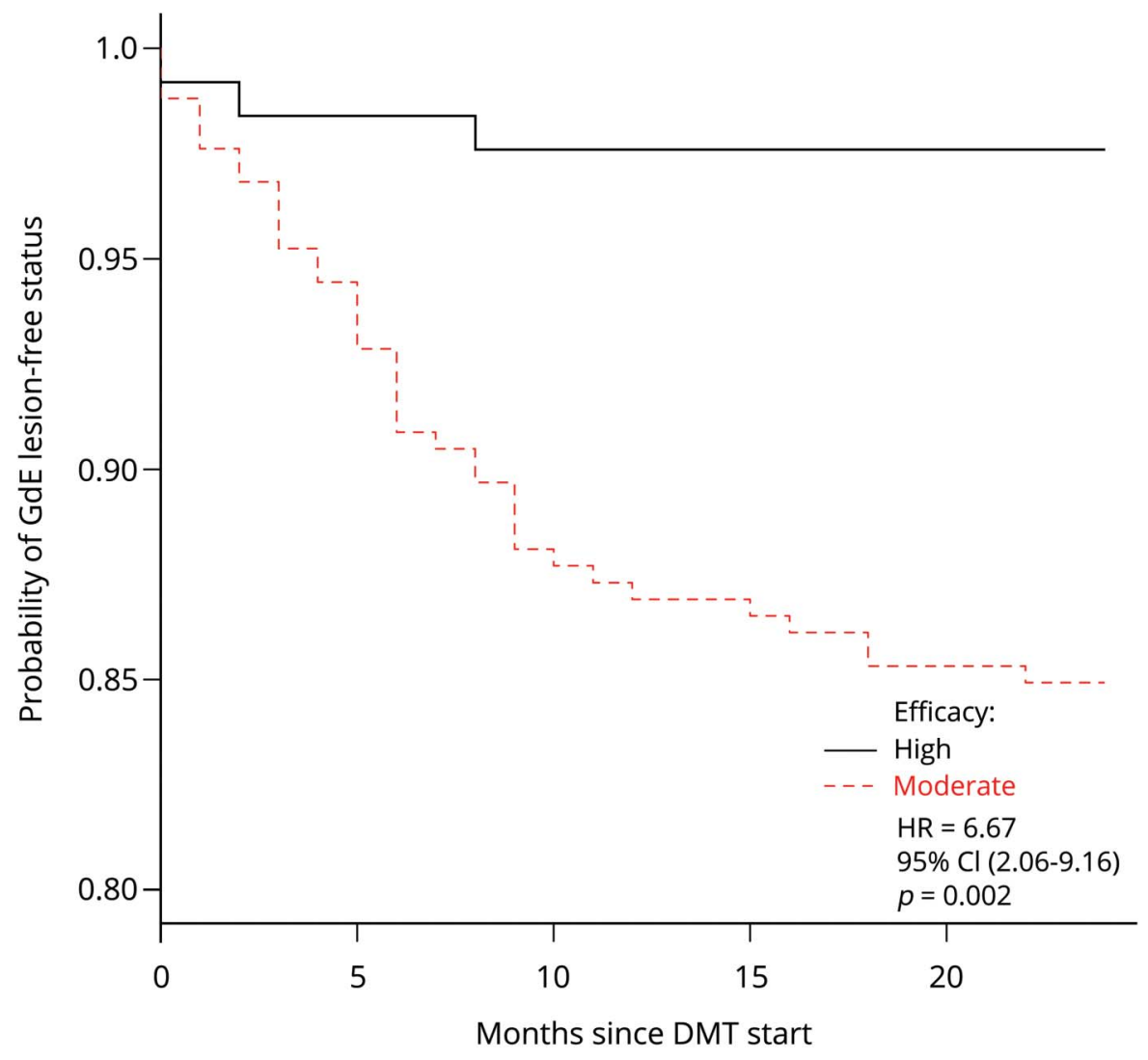

Kaplan-Meier survival analysis showed earlier time to first GdE lesion in patients switching from NTZ to moderate- vs high-efficacy DMT. DMT = disease-modifying therapy. collectively suggest that rituximab and alemtuzumab are favorable options for post-NTZ therapy.

In a subgroup comparative effectiveness analysis stratified by reason for NTZ discontinuation, increased MRI activity in the Mod DMT group was driven by patients who withdrew from NTZ due to breakthrough disease. A similar finding, reporting on relapse rates, was observed in a study by Jokubaitis et al. ${ }^{14}$ that reported that $30 \%$ of patients with disease activity on NTZ relapsed within the first 6 months of transitioning onto fingolimod $(R R=0.001-0.13)$.

Other predictors of disease reactivation following NTZ withdrawal were described. Independent predictors of time to first clinical relapse in NTZ switchers to fingolimod in the Jokubaitis et al. ${ }^{14}$ study were the number of clinical relapses within the previous 6 months $(\mathrm{HR}=1.59 ; p=0.002)$ and washout duration of 2-4 months compared with no washout at all $(\mathrm{HR}=$ 2.10; $p=0.041)$. A smaller French prospective study investigating the effects of switching from NTZ to fingolimod similarly showed a lower risk of relapse $(\mathrm{OR}=0.23 ; p=0.001)$ during a shorter washout period $<3$ months and with less disease activity before NTZ initiation $(p=0.03) .{ }^{11}$

Approximately 20\% of patients relapsed during the first 6 months of fingolimod treatment in a previous study. ${ }^{11}$ In contrast, our patient population experienced a lower risk of clinical relapses within the first 6 months of therapy when switching from NTZ to Mod DMT (11.7\%) and an even lower risk when transitioning onto another HET (8.7\%). This difference was likely related to the shorter mean washout period in our patient population (1.6 months) vs a mean of 4.3 months in the French study. It is unlikely that the inclusion of dimethyl fumarate in the Mod DMT cohort in our investigation yielded a considerable difference in findings relative to other studies reporting on the effectiveness of NTZ to fingolimod transition. However, if the addition of dimethyl fumarate was a differential factor, the anticipation would be for a greater number of relapses in our cohort (rather than fewer), given that no observational studies to date have demonstrated superior effectiveness of dimethyl fumarate compared with fingolimod. Similarly, another study showed that the ARR 1 year after NTZ to dimethyl fumarate transition was lower in those with a washout duration $\leq 3$ months vs $>3$ months (rate ratio 0.49 , 95\% CI [0.26-0.90]). ${ }^{15}$ Overall, published studies collectively demonstrated that a washout period $<3$ months between NTZ and fingolimod or dimethyl fumarate is important in preventing disease reactivation.

In this study, the treating providers likely started patients on NTZ they thought were at highest risk of disease activity. Thus, the fact that individuals in the Mod group fared worse (despite 
Table 4 Unadjusted and adjusted effectiveness outcomes of NTZ switchers to Mod DMT vs HET by 24-month follow-up, stratified by reason for NTZ discontinuation

\begin{tabular}{|c|c|c|c|c|c|c|}
\hline & \multicolumn{3}{|l|}{ Unadjusted } & \multicolumn{3}{|c|}{ Propensity adjusted } \\
\hline & $\begin{array}{l}\text { Odds or } \\
\text { hazards ratio }\end{array}$ & $95 \% \mathrm{Cl}$ & $p$ Value & $\begin{array}{l}\text { Odds or } \\
\text { hazards ratio }\end{array}$ & $95 \% \mathrm{Cl}$ & $p$ Value \\
\hline \multicolumn{7}{|l|}{$\begin{array}{l}\text { NTZ discontinuation due to } \\
\text { breakthrough disease }^{b}\end{array}$} \\
\hline Proportion with clinical relapse & 1.64 & $0.89-4.92$ & 0.083 & 1.71 & $0.95-4.74$ & 0.061 \\
\hline \multicolumn{7}{|l|}{ MRI disease activity (intention-to-treat) } \\
\hline MRI activity (GdE + new T2 lesions) & 1.79 & $0.98-5.23$ & 0.057 & 1.87 & $1.01-5.17$ & $0.049^{c}$ \\
\hline GdE lesions & 1.83 & $0.99-5.44$ & 0.053 & 1.94 & $1.06-5.68$ & $0.043^{c}$ \\
\hline New T2 lesions & 1.81 & $0.98-5.03$ & 0.055 & 1.89 & $1.02-5.24$ & $0.047^{c}$ \\
\hline \multicolumn{7}{|l|}{ MRI disease activity (on-treatment) } \\
\hline MRI activity (GdE + new T2 lesions) & 2.74 & $1.18-6.32$ & $0.028^{c}$ & 2.68 & $1.10-6.33$ & $0.034^{c}$ \\
\hline GdE lesions & 3.57 & $1.48-7.23$ & $0.005^{c}$ & 3.42 & $1.49-7.16$ & $0.006^{c}$ \\
\hline New T2 lesions & 2.23 & $1.09-6.87$ & $0.034^{c}$ & 2.31 & $1.09-6.21$ & $0.027^{c}$ \\
\hline Absence of disease activity ${ }^{a}$ & 0.48 & $0.32-0.83$ & $0.004^{c}$ & 0.39 & $0.21-0.89$ & $0.003^{c}$ \\
\hline \multicolumn{7}{|l|}{ NTZ discontinuation due to safety risks ${ }^{b}$} \\
\hline Proportion with clinical relapse & 1.38 & $0.63-3.93$ & 0.382 & 1.33 & $0.62-3.18$ & 0.823 \\
\hline \multicolumn{7}{|l|}{ MRI disease activity (intention-to-treat) } \\
\hline MRI activity (GdE + new T2 lesions) & 1.66 & $0.94-5.33$ & 0.123 & 1.68 & $0.94-4.26$ & 0.131 \\
\hline GdE lesions & 1.72 & $0.97-4.89$ & 0.092 & 1.73 & $0.97-4.54$ & 0.087 \\
\hline New T2 lesions & 1.63 & $0.91-4.21$ & 0.134 & 1.65 & $0.95-4.72$ & 0.098 \\
\hline \multicolumn{7}{|l|}{ MRI disease activity (on-treatment) } \\
\hline MRI activity (GdE + new T2 lesions) & 2.01 & $1.05-4.29$ & $0.043^{c}$ & 2.08 & $1.05-5.26$ & $0.043^{c}$ \\
\hline GdE lesions & 2.45 & $1.12-5.87$ & $0.041^{c}$ & 2.47 & $1.14-6.01$ & $0.039^{c}$ \\
\hline New T2 lesions & 2.16 & $1.07-5.03$ & $0.042^{c}$ & 2.21 & $1.08-4.58$ & $0.041^{c}$ \\
\hline Absence of disease activity ${ }^{a}$ & 0.61 & $0.32-0.89$ & $0.012^{c}$ & 0.62 & $0.33-0.85$ & $0.011^{c}$ \\
\hline
\end{tabular}

Abbreviations: $\mathrm{Cl}=$ confidence interval; DMT = disease-modifying therapy; GdE = gadolinium enhancing; $\mathrm{HET}$ = high-efficacy therapy; Mod = moderate-efficacy therapy; NTZ = natalizumab.

${ }^{a}$ Absence of disease activity defined as absence of clinical relapses + absence of MRI activity (GdE lesions and/or new T2 lesions).

${ }^{b}$ NTZ discontinuation due to breakthrough disease (Mod DMT $n=40$, HET $\left.n=24\right) ;$ NTZ discontinuation due to safety risks $($ Mod DMT $n=196$, HET $n=78)$.

${ }^{c}$ Statistically significant $p$ value (alpha $=0.05$ ).

likely having less aggressive disease) would support the need to transition most patients to HET after NTZ. The current investigation was limited by various factors. First, our PS model could not adjust for unmeasured covariates or residual biases. To reduce the risk of hidden bias, we included a comprehensive list of covariates believed to be important and representative of those factors essential in determining treatment allocation following NTZ discontinuation. Other limitations inherent in observational studies included the retrospective nature of the investigation, missing data (e.g., radiologic and objective measures of disability progression), and the potential for recall bias in patient-reported relapses. However, the frequency of established visits at our centers (on average every 3-6 months) and the requirement for relapse confirmation by the treating clinician before inclusion into the study accounted for the latter concern. We do not routinely assess EDSS scores during follow-up visits; therefore, surrogate measures of disability progression including $20 \%$ worsening of the T25FW and 9HPT were reported. ${ }^{32}$ Our study is also limited in that it was conducted at two tertiary referral MS centers, which likely differ in patient population and treatment practices compared with other community centers. Despite these limitations, this comprehensive real-world study provides clinical insights into the comparative effectiveness of switching from NTZ to alternative treatment strategies that are increasingly used in routine practice. 


\section{Conclusion}

The current study provides real-world clinical insights into DMT switching patterns following NTZ discontinuation. Patients switching from NTZ to Mod DMT vs HET are at relatively increased risk of disease activity within the first 6 months of NTZ withdrawal, which is sustained at 24 months, yielding greater disability progression. The current study also demonstrates that switching to a DMT of at least moderate efficacy following NTZ discontinuation is effective in reducing the risk of rebound disease when restricting the washout period to $<3$ months, particularly in those switching to HET. Thus, these data suggest that an HET should be considered in the appropriate clinical setting when transitioning a patient off of NTZ, especially when due to breakthrough disease. A larger, multicenter study investigating the short- and long-term effects of post-NTZ DMT sequencing is planned to improve upon the external validity of the current findings.

\section{Acknowledgment}

The authors thank the patients treated at the Cleveland Clinic Lou Ruvo Center for Brain Health and Cleveland Clinic Mellen Center.

\section{Study funding}

This study received partial funding support from the Sheila and Eric Samson Foundation.

\section{Disclosure}

C.M. Hersh has received speaking and consulting fees from Genzyme, Genentech, EMD Serono, Novartis, and Biogen and has received research support paid to her institution from Biogen, Genentech, and PCORI. H. Harris reports no disclosures. D. Conway has received research support paid to his institution from Novartis and the National Multiple Sclerosis Society and has received personal compensation for consulting from Novartis and Tanabe Laboratories. L.H. Hua has received speaking and consulting fees from Biogen, Genzyme, Genentech, Novartis, Celgene, and EMD Serono. Full disclosure form information provided by the authors is available with the full text of this article at Neurology.org/cp.

\section{Publication history}

Received by Neurology: Clinical Practice September 23, 2019. Accepted in final form December 9, 2019.

Appendix Authors

\begin{tabular}{lll}
\hline Name & Location & Contribution \\
\hline $\begin{array}{l}\text { Carrie M. } \\
\text { Hersh, DO, MSc }\end{array}$ & $\begin{array}{l}\text { Cleveland Clinic Lou Ruvo } \\
\text { Center for Brain Health }\end{array}$ & $\begin{array}{l}\text { Drafted and revised the } \\
\text { manuscript for intellectual } \\
\text { content and contributed to } \\
\text { the study concept, design, } \\
\text { acquisition, analysis, and } \\
\text { interpretation of the data }\end{array}$ \\
$\begin{array}{lll}\text { Haleigh Harris, } \\
\text { BA }\end{array}$ & $\begin{array}{l}\text { Cleveland Clinic Lou Ruvo } \\
\text { Center for Brain Health }\end{array}$ & $\begin{array}{l}\text { Contributed to the data } \\
\text { acquisition }\end{array}$ \\
\hline
\end{tabular}

\section{Appendix (continued)}

\begin{tabular}{lll}
\hline Name & Location & Contribution \\
\hline $\begin{array}{l}\text { Devon } \\
\text { Conway, MD }\end{array}$ & $\begin{array}{l}\text { Cleveland Clinic Mellen } \\
\text { Center }\end{array}$ & $\begin{array}{l}\text { Contributed to the study } \\
\text { concept, interpretation of } \\
\text { the data, and revision of the } \\
\text { manuscript }\end{array}$ \\
\hline Le H. Hua, MD & $\begin{array}{l}\text { Cleveland Clinic Lou Ruvo } \\
\text { Center for Brain Health }\end{array}$ & $\begin{array}{l}\text { Contributed to the study } \\
\text { concept, interpretation of } \\
\text { the data, and revision of the } \\
\text { manuscript }\end{array}$ \\
\hline
\end{tabular}

\section{References}

1. Polman $\mathrm{CH}, \mathrm{O}$ 'Connor PW, Havrdova E, et al. A randomized, placebo-controlled tria of natalizumab for relapsing multiple sclerosis. N Engl J Med 2006;354:899-910.

2. Miller DH, Soon D, Fernando KT, et al. MRI outcomes in a placebo-controlled trial of natalizumab in relapsing MS. Neurology 2007;68:1390-1401.

3. Merkel B, Butzkueven H, Traboulsee AL, Havrdova E, Kalincik T. Timing of highefficacy therapy in relapsing-remitting multiple sclerosis: a systematic review. Autoimmun Rev 2017;16:658-665.

4. Bloomgren G, Richman S, Hotermans C, et al. Risk of natalizumab-associated progressive multifocal leukoencephalopathy. N Engl J Med 2012;366:1870-1880.

5. Plavina T, Subramanyam M, Bloomgren G, et al. Anti-JC virus antibody levels in serum or plasma further define risk of natalizumab-associated progressive multifocal leukoencephalopathy. Ann Neurol 2014;76:802-812.

6. Gueguen A, Roux P, Deschamps R, et al. Abnormal inflammatory activity returns after natalizumab cessation in multiple sclerosis. J Neurol Neurosurg Psychiatry 2014;85:1038-1040.

7. O'Connor PW, Goodman A, Kappos L, et al. Disease activity return during natalizumab treatment interruption in patients with multiple sclerosis. Neurology 2011;76:1858-1865.

8. Vellinga MM, Castelijns JA, Barkof F, et al. Postwithdrawal rebound increase in T2 lesional activity in natalizumab-treated MS patients. Neurology 2008;70:1150-1151.

9. Papeix C, Vukusic S, Casey R, et al. Risk of relapse after natalizumab withdrawal: results from the French TYSEDMUS cohort. Neurol Neuroimmunol Neuroinflamm 2016;3:e297.

10. Sellner J, Rommer PS. A review of the evidence for a natalizumab exit strategy for patients with multiple sclerosis. Autoimmun Rev 2019;18:255-261.

11. Cohen M, Maillart E, Tourbah A, et al. Switching from natalizumab to fingolimod in multiple sclerosis: a French prospective study. JAMA Neurol 2014;71:436-441.

12. Fox RJ, Cree BAC, De Seze J, et al. MS disease activity in RESTORE: a randomized 24-week natalizumab interruption study. Neurology 2014;82:1491-1498.

13. Kappos L, Radue EW, Comi G, et al. Switching from natalizumab to fingolimod: a randomized, placebo-controlled study in RRMS. Neurology 2015;85:29-39.

14. Jokubaitis VG, Li V, Kalincik T, et al. Fingolimod after natalizumab and the risk of short-term relapse. Neurology 2014;82:1204-1211.

15. Cohan SL, Moses H, Calkwood J, et al. Clinical outcomes in patients with relapsing remitting multiple sclerosis who switch from natalizumab to delayed-release dimethyl fumarate: a multicenter retrospective observational study (STRATEGY). Mult Scle Relat Disord 2018;22:27-34.

16. Calabrese M, Pitteri M, Farina G, et al. Dimethyl fumarate: a possible exit strategy from natalizumab treatment in patients with multiple sclerosis at risk for severe adverse events. J Neurol Neurosurg Psychiatry 2017;88:1073-1078.

17. Zurawski J, Flinn A, Sklover L, Sloane JA. Relapse frequency in transitioning from natalizumab to dimethyl fumarate: assessment of risk factors. J Neurol 2016;263:1511-1517.

18. Edwards KR, Kamath V, O'Connor J. Switching From Natalizumab to Teriflunomide in Multiple Sclerosis Patients Who Have Received Natalizumab for More Than 12 Months. Abstract presented at 2014 CMSC ACTRIMS Annual Meeting; May 29, 2014; Dallas, TX. Available at: cmsc.confex.com $/ \mathrm{cmsc} / 2014 /$ webprogram $/ \mathrm{Pa}$ per2464.html. Accessed July 16, 2019.

19. Gobbi C, Meier DS, Cotton F, et al. Interferon beta $1 \mathrm{~b}$ following natalizumab discontinuation: one year, randomized, prospective, pilot trial. BMC Neurol 2013;13:101.

20. Havla J, Gerdes LA, Meinl I, et al. De-escalation from natalizumab in multiple sclerosis: recurrence of disease activity despite switching to glatiramer acetate. J Neurol 2011;258:1665-1669.

21. Rossi S, Motta C, Studer V, et al. Effect of glatiramer acetate on disease reactivation in MS patients discontinuing natalizumab. Eur J Neurol 2013;20:87-94.

22. Mancinelli CR, Scarpazza C, Santuccio G, et al. Dealing with highly active multiple sclerosis after natalizumab-associated PML: could rituximab be of help? Neurol Sci 2018;39:965-966.

23. Malucchi S, Capobianco M, Lo Re M, et al. High-risk PML patients switching from natalizumab to alemtuzumab: an observational study. Neurol Ther 2017;6:145-152.

24. Rosenbaum R, Rubin DB. The central role of the propensity score in observational studies for causal effects. Biometrika 1983;70:41-55.

25. Hersh CM, Love TE, Bandyopadhyay A, et al. Comparative efficacy and discontinuation of dimethyl fumarate and fingolimod in clinical practice at 24-month follow-up. Mult Scler J Exp Transl Clin 2017;3:2055217317715485

26. Vollmer B, Nair KV, Sillau SH, Corboy J, Vollmer T, Alvarez E. Comparison of fingolimod and dimethyl fumarate in the treatment of multiple sclerosis: two-year experience. Mult Scler J Exp Transl Clin 2017;3:2055217317725102.

27. Vollmer B, Ontaneda D, Bandyopadhyay A, et al. Discontinuation and comparative effectiveness of dimethyl fumarate and fingolimod in 2 centers. Neurol Clin Pract 2018;8:292-301. 
Fox RJ, Chan A, Zhang A, et al. Comparative effectiveness using a matching-adjusted indirect comparison between delayed-release dimethyl fumarate and fingolimod for the treatment of multiple sclerosis. Curr Med Res Opin 2017;33:175-183.

29. Prosperini L, Lucchini M, Haggiag S, et al. Fingolimod vs dimethyl fumarate in multiple sclerosis. A Real-World Propensity Score-Matched Study. Neurology 2018; 91:e153-e161.

30. Wicks P, Rasouliyan L, Katic B, Nafees B, Flood E, Sasané R. The real-world patient experience of fingolimod and dimethyl fumarate for multiple sclerosis. BMC Res Notes 2016;9:434.

31. Rudick RA, Cutter G, Reingold S. The multiple sclerosis functional composite: a new clinical outcome measure for multiple sclerosis trials. Mult Scler 2006;8:359-365.

32. Kragt JJ, van der Linden FA, Nielsen JM, Uitdehaag BM, Polman CH. Clinical impact of $20 \%$ worsening on timed 25 -foot walk and 9-hole peg test in multiple sclerosis. Mult Scler 2006;12:594-598.

33. Kroenke K, Spitzer RL, Williams JB. The PHQ-9: validity of a brief depression severity measure. J Gen Intern Med 2001;16:606-613.

34. Schwartz CE, Vollmer T, Lee H. Reliability and validity of two self-report measures of impairment and disability for MS. North American Research Consortium on Multiple Sclerosis Outcomes Study Group. Neurology 1999;52:63-70.
35. Team RC. R: A Language and Environment for Statistical Computing. Vienna: R Foundation for Statistical Computing; 2014

36. Rubin DB. Using propensity scores to help design observational studies: application to the tobacco litigation. Health Serv Outcomes Res Methodol 2001;2 169-188.

37. Plavina T, Muralidharan KK, Kuesters G, et al. Reversibility of the effects of natalizumab on peripheral immune cell dynamics in MS patients. Neurology 2017;89: $1584-1593$.

38. Cree B, de Seze J, Fox R, et al. Natalizumab effects during a 6 month dose interruption: relationship of pharmacokinetic (PK), pharmacodynamic (PD) and MRI measurement. Neurology 2013;80:S41.003.

39. Miravalle A, Jensen R, Kinkel RP. Immune reconstitution inflammatory syndrome in patients with multiple sclerosis following cessation of natalizumab therapy. Arch Neurol 2011;68:186-191.

40. Villaverde-Gonzalez R, Gracia Gil J, Perez Sempere A, et al. Observational study of switching from natalizumab to immunomodulatory drugs. Eur Neurol 2017;77: 130-136.

41. Comi G, Gold R, Dahlke F, et al. Relapses in patients treated with fingolimod after previous exposure to natalizumab. Mult Scler 2015;21:786-790. 


\section{Neurology ${ }^{\circ}$ Clinical Practice}

\section{Effect of switching from natalizumab to moderate- vs high-efficacy DMT in clinical practice}

Carrie M. Hersh, Haleigh Harris, Devon Conway, et al.

Neurol Clin Pract published online February 12, 2020

DOI 10.1212/CPJ.0000000000000809

This information is current as of February 12, 2020

$\begin{array}{ll}\begin{array}{l}\text { Updated Information \& } \\ \text { Services }\end{array} & \begin{array}{l}\text { including high resolution figures, can be found at: } \\ \text { http://cp.neurology.org/content/early/2020/02/12/CPJ.0000 } \\ \text { 09.full.html }\end{array} \\ \text { This article has been cited by 1 HighWire-hosted articles: } \\ \text { http://cp.neurology.org/content/early/2020/02/12/CPJ.0000 } \\ \text { 09.full.html\#\#otherarticles } \\ \text { This article, along with others on similar topics, appears } \\ \text { following collection(s): } \\ \text { Multiple sclerosis } \\ \text { http://cp.neurology.org//cgi/collection/multiple_sclerosis } \\ \text { Information about reproducing this article in parts (figures, } \\ \text { its entirety can be found online at: } \\ \text { http://cp.neurology.org/misc/about.xhtml\#permissions } \\ \text { Information about ordering reprints can be found online: } \\ \text { http://cp.neurology.org/misc/addir.xhtml\#reprintsus }\end{array}$

Updated Information \& Services

\section{Citations}

\section{Subspecialty Collections}

Permissions \& Licensing

Reprints including high resolution figures, can be found at:

http://cp.neurology.org/content/early/2020/02/12/CPJ.00000000000008

This article has been cited by 1 HighWire-hosted articles: http://cp.neurology.org/content/early/2020/02/12/CPJ.00000000000008 .rull.html\#\#) herarticles

This article, along with others on similar topics, appears in the following collection(s)

Multiple sclerosis

isclerosis

(figures,tables) or in its entirety can be found online at:

Information about ordering reprints can be found online:

http://cp.neurology.org/misc/addir.xhtml\#reprintsus

Neurol Clin Pract is an official journal of the American Academy of Neurology. Published continuously since 2011, it is now a bimonthly with 6 issues per year. Copyright Copyright @ 2020 The Author(s). Published by Wolters Kluwer Health, Inc. on behalf of the American Academy of Neurology.. All rights reserved. Print ISSN: 2163-0402. Online ISSN: 2163-0933.

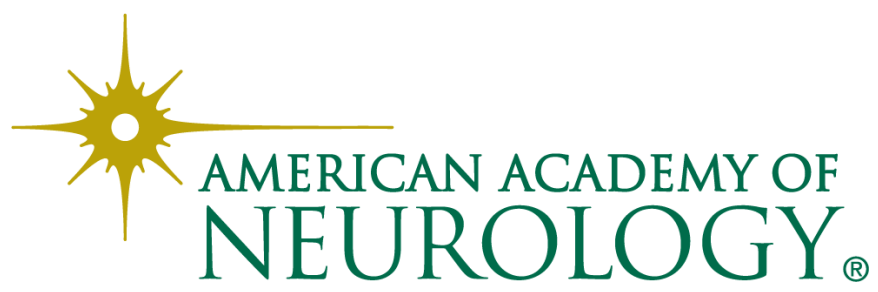

